\title{
Diseño Y Validación Del Instrumento Estrigeree Para La Evaluación Del Estrés En Prácticas Clínicas De Fisioterapia
}

\author{
María Guadalupe Becerra Guevara, (PSS. Enf.) \\ María Virginia Gómez Márquez, (PSS. Enf.) \\ Rosa Isela Lugo Hernández, (PSS. Enf.) \\ Gustavo Argenis Hernández Segura, (M.I.M.) \\ Universidad Autónoma de Querétaro, Mexico
}

Doi:10.19044/esj.2018.v14n23p57 URL:http://dx.doi.org/10.19044/esj.2018.v14n23p57

\begin{abstract}
Background: Stress represents a problem in multiple spheres of the person such as psychological, emotional, physical, and social among others; however, stress in academic life does not receive enough attention in scientific evidence. Purpose: was to design and validate an instrument that allows to assess the level of stress in students of clinical physiotherapy practices. The instrument was called Estrigeree. Methods: It is study that was developed in two phases which are included in the design and validation of an instrument, the validation was characterized by an expert review, a pilot test application of the Test / Retest type and a final application. A sample of $n=10$ was used for the pilot test and $\mathrm{n}=100$ for the final test with an average age of 52.19. The percentage of men was $28 \%$ and $72 \%$ for women. Results: In the pilot test for the test, the Cronbach's alpha was 0.87 , for the Re-test it was 0.62 points. In the final test it was observed that $2.9 \%$ of the population did not present stress, $34.3 \%$ reported low stress, $52 \%$ showed a moderate level and $10.8 \%$ of the population reported a lot of stress. Conclusions: The instrument is valid and reliable, its measurement is stable, it is clear, systematic and complete, of rapid application and processing. Finally, it fulfills its design objective.
\end{abstract}

Keywords: Design and validation, stress, clinical practice at physical therapy

\section{Resumen}

Introducción: El estrés representa una problemática en múltiples esferas de la persona tales como la psicológica, emocional, física, social, entre otras; sin embargo el estrés en la vida académica no recibe la suficiente 
atención en la evidencia científica. Objetivo: diseñar y validar un instrumento que permita evaluar el nivel de estrés en estudiantes de prácticas clínicas de fisioterapia. El instrumento fue denominado Estrigeree. Metodología: se trata de un estudio que se desarrolló en dos fases las cuales constan en el diseño y la validación de un instrumento, esta última se caracterizó por una revisión de expertos, una aplicación de prueba piloto de tipo Test / Re-test y una aplicación final. Se utilizó una muestra de $n=10$ para la prueba piloto y de $\mathrm{n}=100$ para la prueba final con una media de edad de 52.19. El porcentaje de hombres fue de $28 \%$ y del $72 \%$ para mujeres.

Resultados: en la prueba piloto para el Test, el alfa de Cronbach resultó de 0.87, para el Re-test fue de 0.62 puntos. En la prueba final se observó que el $2.9 \%$ de la población no presentó estrés, el 34.3\% refirió poco estrés, el 52\% arrojó un nivel moderado y el 10.8\% de la población refirió mucho estrés.

Conclusiones: El instrumento es válido y confiable, es estable es su medición temporal, es claro, sistemático y completo, de rápida aplicación $\mathrm{y}$ procesamiento. Finalmente, cumple con su objetivo de diseño.

Palabras Clave: Diseño y validación, estrés, prácticas clínicas de fisioterapia

\section{Introducción:}

Actualmente el estrés representa una problemática que requiere una atención cada vez mayor ya que dicho fenómeno va en aumento debido a los cambios sociales y económicos del país, sin embargo, en la vida académica no recibe el suficiente análisis desde la perspectiva científica ya que existen múltiples factores con un nivel potencialmente estresante que impactan directamente en el rendimiento académico de los estudiantes (Domínguez, Domínguez y Guerrero, 2015).

Alfonso, Calcines, Monteagudo y Nieves (2015), en el estudio "Estrés académico" citando a Díaz Martín (2014), "refieren que desde los grados preescolares hasta la educación universitaria de posgrado, cuando una persona está en periodo de aprendizaje experimenta tensión. A esta se le denomina estrés académico y ocurre tanto en el estudio individual como en el aula".

Diversos estudios señalan que los factores estresantes que más afectan a los estudiantes en las prácticas, son los relacionados con el desconocimiento, impotencia e incertidumbre ante una situación, el aumento de la carga de trabajo, falta de tiempo y algunos aspectos del entorno (Rodríguez, Sanmiguel, Muñoz y Rodríguez, 2014).

Los centros de práctica brindan la oportunidad de enfrentarse a situaciones donde se tiene la posibilidad de desempeñarse con la responsabilidad de un profesional siendo un estudiante, dándole la posibilidad de realizar en escenarios reales todo lo visto en las aulas previamente; estos aprendizajes prácticos refuerzan sus conocimientos por lo que las prácticas 
formativas permiten relacionarse con un medio laboralmente exigente $\mathrm{y}$ proyectarse social y laboralmente (Tamayo, Ortiz y Giraldo 2014).

El entorno sanitario proporciona muchos estímulos generadores de estrés debido al contacto con las enfermedades, el dolor, el sufrimiento, la muerte de los pacientes o el hecho de tener que desempeñar un nuevo rol para el que aún no se está completamente preparado (Gómez, Puga, Mayan y Gandoy, 2012).

La Fisioterapia es una profesión dinámica con una base teórica establecida y aplicaciones clínicas extendidas, particularmente en la preservación, el desarrollo y restauración máxima de las funciones físicas, por lo que un fisioterapeuta debe demostrar competencia profesional en sus diferentes campos de actuación, expresando conocimientos, habilidades y actitudes en actividades de promoción, mantenimiento, prevención, protección y recuperación de la Salud. Así mismo tiene el objetivo de preservar, desarrollar y restaurar la integridad de los órganos, los sistemas y las funciones, siendo un profesional volcado en el desarrollo científico y apto para adquirir conocimientos que puedan garantizar una atención a la salud de forma continuada y permanente (World Confederation for Physical Therapy, 2011).

En el estudio publicado por Gutiérrez, Becerra, Martínez y García (2017) se observa que incluso en estudiantes de primer semestre de Fisioterapia, los cuales aún no desarrollan incluso prácticas clínicas, están inmersos en niveles de estrés de moderado a grave intensidad.

Para llevar a cabo el desarrollo, ejecución, y evaluación de una investigación adecuada se requiere de la aplicación de instrumentos de acuerdo al tema sometido a estudio, lo que deriva la importancia de la creación de los instrumentos confiables y precisos para que la obtención de información sea clara y específica (Baptista, Hernández y Fernández, 2014).

El instrumento con mayor reproducibilidad para la evaluación del estrés es el Maslach Burnout Test el cual fue creado por Christina Maslach en 1980 en donde se asocia al estrés de manera laboral, sin embargo a lo largo de la investigación sobre este tema se ha observado la existencia de una mezcla de términos relacionados que en ocasiones se utilizan indistintamente, como es el caso de estrés, burnout y depresión. Por otra parte, los estudios no parecen hacer un gran esfuerzo por establecer un límite claro, sino que prefieren apoyarse en la información ya existente en otras áreas para ahondar más en la promoción de la salud y la prevención de los problemas laborales (Martínez, 2010).

Otro de los instrumentos que se ha utilizado es la Escala Unidimensional Burnout Estudiantil (EUBE), que fue aplicada por primera vez para medir el nivel de Burnout en los estudiantes de medicina. Esta escala, creada y validada por el Dr. C. Arturo Barraza y colaboradores (2008) de la 
Universidad Pedagógica de Durango, consta de 15 ítems y reportó un nivel de confiabilidad de 91 en alfa de Cronbach y de 90 en la confiabilidad por mitades según la fórmula de Spearman-Brown. Este instrumento mide el nivel de agotamiento físico, emocional y cognitivo en los estudiantes, respetando el concepto unidimensional del Síndrome de Burnout por el que se rige este trabajo y ha demostrado un muy buen nivel de confiabilidad. Además se ha confirmado la homogeneidad y unidireccionalidad de los ítems que componen la escala y los resultados en la relación de los ítems y el puntaje global de la escala permiten afirmar que todos los ítems forman parte del Burnout Estudiantil (Rosales, 2012).

\section{Metodología:}

Se llevó a cabo un estudio de tipo diseño y validación de un instrumento capaz de medir el estrés en una población de estudiantes de fisioterapia que se encuentran cursando la práctica clínica, lo anterior se fundamentó en el método hipotético deductivo. El objetivo de la investigación fue conocer si el instrumento es válido y confiable para la evaluación del estrés en estudiantes de prácticas clínica de fisioterapia.

\section{a) Diseño de instrumento}

Se consideró importante la creación de un instrumento ex profeso que contuviera los constructos relacionados a las situaciones que puedan desarrollar más estrés en estudiantes de fisioterapia en prácticas clínicas. Dicho instrumento llevó por nombre "Estrigeree" (Imagen 1) el cual deriva del griego y significa provocar tensión. El diseño del instrumento se realizó en las etapas de valoración de la problemática, revisión de la literatura, planificación, elaboración de los constructos, elaboración de las dimensiones así como de los ítems, análisis y confección matemática de los mismos y finalmente una evaluación por expertos. 
Imagen 1. Carátula frontal de evaluación del instrumento Estrigeree.

Folio:

Cuestionario Estrigeree
Estrigeree es un instrumento de auto aplicación y heteroaplicación que está orientado a conocer el nivel de estrés en los estudiantes de fisioterapia en prácticas clínicas.
Instrucción 1:
Favor de leer atentamente las preguntas que se formulan en cada apartado y contesta de acuerdo a tu criterio, posteriormente realiza la sumatoria para obtener los puntajes finales. Se
evaluarán tres dimensiones, cada una conformada por diferentes factores, como son: alimentación, sueño, gastos económicos y relaciones interpersonales; los calificadores a utilizar se
encuentran en cada factor a evaluar.
Nota: en el factor de actividad física no aplica una evaluación numérica para obtención de puntaje, solo funciona como retroalimentación.

\begin{tabular}{|c|c|c|c|}
\hline \multicolumn{4}{|c|}{ Datos sociodemográficos } \\
\hline Edad: & Sexo: & Semestre: & Práctica No.: \\
\hline
\end{tabular}

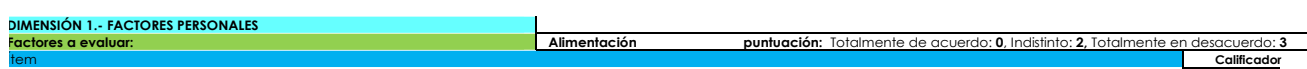

1.- Considero que mi alimentación es de buena calidad en relación a cantidad, con base en la recomendación de hacer 5 comidas al día. 2.- Considero que mi alimentación diaria es buena en relación a calidad, con base en la recomendación de incluir los tres grupos de alimentos (frutas y verduras, cereales, eguminosas y alimentos de origen animal).

3.- Me hidrato adecuadamente, tomando de 2 a 3 litros de agua al día.

4.- Evito consumir comida chatarra y comida rápida tales como: (frituras, embutidos etc.)

5.- Las condiciones de la práctica clínica (carga de trabajo, horarios, distancia, etc.) me permiten consumir el/los alimento(s) saludable (s).

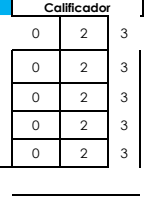

Factores a evaluar: Sueño puntuación: Totalmente de acuerdo: 3, Indistinto: 2, Totalmente en desacuerdo: 1 .

6.-Considero que la calidad de mi sueño no es la óptima (entre 6-7 horas de descansol

7.- Me desvelo constantemente.

8.- Sufro de insomnio.

9.- Me cuesta despertarme.

10.- Me siento somnoliento (a) durante las prácticas.

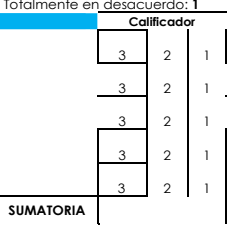

\begin{tabular}{|c|}
\hline Factores a evaluar \\
\hline
\end{tabular}

11.- Me he sentido presionado por los gastos de alguna materia.

12.- He tenido que gastar más de lo estimado por algún imprevisto en alguna materic.

13.- He tenido que pedir dinero prestado para pagar los gastos de la escuela.

14.- He pensado en dejar la escuela y ponerme a trabajar.

15.- He faltado a clases para generar dinero.

\begin{tabular}{|c|c|c|}
\hline Factores a evaluar: & & \\
\hline Si tu respuesta anterior es NO, pasa al ítem 18 & & \\
\hline 17.- Cuando practico deporte percibo que disminuye mi nivel de estrés. & Si & No \\
\hline 19.- Practico alguna actividad recreativa. そ̇Cuálę: & Si & No \\
\hline Si tu respuesta anterior es NO, pasa al ítem 21 & & \\
\hline 20.- Cuando practico alguna actividad recreativa percibo que disminuye mi nivel de estrés. & Si & No \\
\hline
\end{tabular}

DIMENSIÓN 2.- FACTORES INTERPERSONALES

\begin{tabular}{l|l} 
DIMENSIÓN 2.- FACTORES INTERPERSONALES & Interpersonal \\
factores a evaluar: &
\end{tabular}

puntuación: Totalmente de acuerdo: $\mathbf{0}$, Indistinto: $\mathbf{2}$, Totalmente en desacuerdo: 3

22.- Mi familia me apoya ante situaciones difíciles de las prácticas.

23.- La relación con mis compañeros es óptima.

24.- Recibo apoyo de mis compañeros cuando me enfrento a situaciones escolares dificiles.

25.- La relación con mi actual asesor de prácticas es buena.

26.- Recibo apoyo de mi asesor de prácticas en situaciones dificiles.

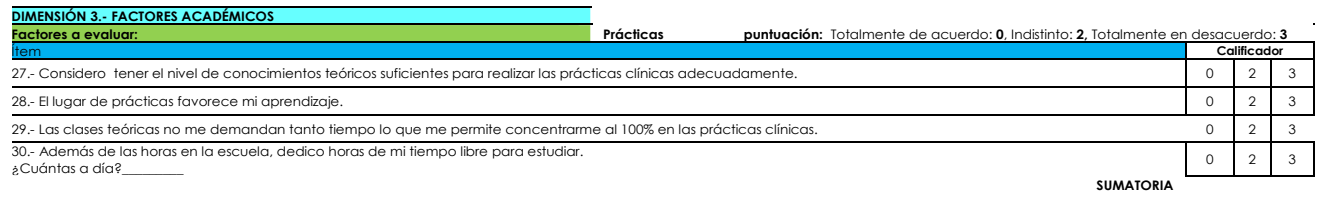

Fuente: instrumento Estrigeree 
En la valoración de la problemática se realizó a través de la identificación de las necesidades de acuerdo a la experiencia de los estudiantes de fisioterapia ante las prácticas clínicas, la hipótesis, los objetivos y las pregunta de investigación. La revisión de la literatura científica se llevó a cabo por medio de varios motores de búsqueda en donde se encontraron instrumentos para medir el estrés enfocados hacia estudiantes universitarios en general, estudiantes de medicina, estudiantes de enfermería y sólo una referencia acerca del estrés en estudiantes de fisioterapia previamente citado. Posteriormente se dio a la tarea de planificar un instrumento que permitiera valorar a través de ítems de acuerdo a los factores asociados al rendimiento académico en estudiantes universitarios (Garbanzo, 2007).

Tal instrumento consta de 4 dimensiones que están enfocados a factores sociodemográficos, personales, interpersonales y académicos del estudiante en etapa universitaria que podrán alterar o mejorar el aprovechamiento de cada individuo. La elaboración de los ítems se comenzó con el desarrollo de algunas interrogantes que ayudarán a identificar las principales necesidades de los estudiantes universitarios, dando continuidad específicamente con los estudiantes de fisioterapia y sus factores asociados al rendimiento académico.

Después de tomar la decisión de qué tipo de preguntas se seleccionarían, el número de preguntas, el valor ponderal de cada una; se dio a la tarea de redactarlas, las cuales tuvieron que ser claras y comprensibles para los encuestados adaptando el lenguaje a las características de los entrevistados. Fue necesario también elaborar preguntas específicas para cada una de las dimensiones que se iban a medir con la finalidad de evitar que se confundieran con su contenido.

\section{b) Confiabilidad y validez}

Un instrumento de medición debe contar con los requisitos de validez y confiabilidad, para que sea el adecuado en la recolección de datos. La validez se refiere al grado en que una prueba proporciona información que es apropiada a la decisión que se toma y responde a la pregunta, es decir, ¿Con qué fidelidad corresponde el universo o población al atributo que se va a medir?, mientras que la confiabilidad tiene que ver con la exactitud y precisión del procedimiento de medición (Corral, 2009).

\section{c) Validación por expertos}

La estructura y confección de los ítems que conforman todo el instrumento fue sometido a un proceso de validación por criterios de expertos para evaluar la validez del constructo de los ítems en relación con la definición operacional y las categorías propuestas con base en la pertinencia, claridad y validez, es decir, los ítems y las dimensiones miden lo que tienen que medir, 
si el lenguaje es claro y universal, y si técnicamente la elaboración es correcta (Lewis, 2003).

Las características de dichos expertos se destacaron por tener al menos dos años en el área de investigación, contar con grado de maestría o especialidad, al menos dos expertos con perfiles de fisioterapia, un experto en estadística, así como al menos un experto en investigación cuali-cuantitativa y un experto en psicología.

\section{d) Asignación de puntaje para el instrumento}

Para la asignación de puntaje se tomó como referencia la escala de la Clasificación Internacional del Funcionamiento de la Discapacidad y de la salud, conocida como CIF (OMS, 2001). De acuerdo a lo anterior se estableció una escala de tipo Likert con 5 niveles determinados como nulo, poco, moderado, mucho y total los cuales permitieron por su puntaje poder medir y establecer el nivel de estrés en que se encuentra el estudiante al concluir el instrumento.

\section{e) Procedimiento de validación}

Se presentó el instrumento Estrigeree ante los expertos que se eligieron de acuerdo a los requisitos previamente establecidos. Se desarrolló una breve exposición para dar a conocer el objetivo así como el material asignado para su validación siendo. Se procedió con las instrucciones para la evaluación de los ítems, posteriormente se analizó la pertinencia, claridad y validez; se continuó con la asignación del porcentaje de validación por cada uno de ellos, finalmente los expertos expusieron sus observaciones y sugerencias para la mejora del instrumento.

\section{f) Prueba piloto}

El instrumento fue sometido a un pilotaje considerado pre-test para evaluar la aplicabilidad y viabilidad del mismo a los estudiantes, los cuales recibieron información previa acerca del estudio y así como del consentimiento informado. Para el proceso de validación y evaluación de la confiabilidad interna, se consideró como pos-test una segunda aplicación 4 semanas después del primer test. La consistencia interna se determinó a través del cálculo del coeficiente Alfa de Cronbach de forma global, eliminando el puntaje del ítem a analizar y con la correlación Ítem-total así como con el coeficiente de determinación (George y Mallery, 2003).

\section{Resultados:}

Previo a la validación por expertos el instrumento se compuso de 5 dimensiones y 36 ítems. Como resultado de la evaluación y el exhaustivo análisis de los mismos, finalmente se obtuvo el producto de un instrumento 
que contiene 3 dimensiones denominadas factores personales, factores interpersonales y factores académicos.

En la dimensión de los factores personales se lograron integrar un total de 21 ítems; 5 ítems para la dimensión de factores interpersonales, así como 4 ítems para la dimensión de los factores académicos. Dicha disparidad en la cantidad de ítems contenidos en cada dimensión, sugiere el uso de la fórmula del promedio ponderado (Baptista, Hernández y Fernández, 2014) para asignar los valores que permitieron evaluar tanto el contenido de toda la dimensión, los subgrupos que las conforman y a su vez el gran total, así como también el adecuado análisis a través del Alpha de Cronbach el cual se realizó en una prueba piloto razonada como el Test en donde se recabó la información para la medición de la confiabilidad obteniendo como resultando la puntuación del instrumento de 0.87 , considerado en general como un grado de confiabilidad alto, mientras que en el Re-Test se aplicó con la muestra del estudio, en donde se obtuvo un resultado de 0.62 puntos, considerado como suficiente.

El estudio se realizó a un total de $n=102$ alumnos donde el $28 \%$ fueron hombres y el $72 \%$ fueron mujeres, con un promedio de edad de 21.64 y una desviación estándar de SD 1.3. Todos ellos distribuidos en cuatro diferentes prácticas clínicas.

Con base en lo anterior y tal como se puede observar en la Imagen 2, se desarrolló una tabla de descarga de puntos en la cual se establecen los valores mínimos y máximos distribuidos en quintiles tanto de los subgrupos, de las dimensiones propiamente así como del gran total, lo que permite otorgar una interpretación del nivel de estrés correspondientemente tanto para el encuestado así como para el evaluador. Dicho nivel de estrés fue determinado y justificado por la fórmula del promedio ponderado en donde lo que interesa en asignar límites superiores e inferiores del comportamiento numérico de los ítems independientemente de la cantidad que componen a los subgrupos o dimensiones a evaluar. 
Imagen 2. Carátula posterior de instrucciones para la tabla de descarga de puntos e interpretación del nivel de estrés.

Instrucción 2:

A continuación se muestran las tablas de descarga en donde podrás evaluar tu nivel de estrés por factor, por dimensión y en general colocando tus puntajes obtenidos en cada tabla respectivamente y cotejando tus resultados con los establecidos en cada tabla de referencia tal y como se muestra en el siguiente ejemplo:

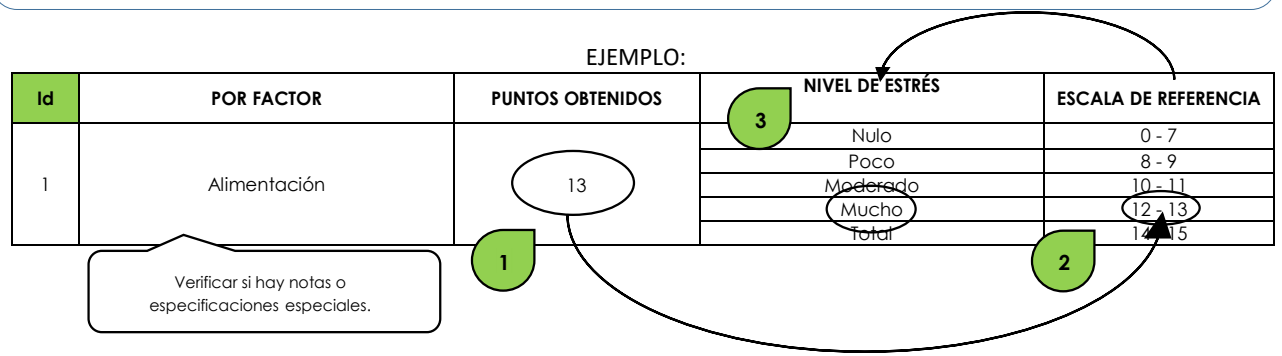

\begin{tabular}{|c|c|c|c|c|}
\hline Id & POR FACTOR & PUNTOS OBTENIDOS & NIVEL DE ESTRÉS & $\begin{array}{l}\text { ESCALA DE } \\
\text { REFERENCIA }\end{array}$ \\
\hline \multirow{5}{*}{1} & \multirow{5}{*}{ Alimentación } & & Nulo & $0-7$ \\
\hline & & & Poco & $8-9$ \\
\hline & & & Moderado & $10-11$ \\
\hline & & & Mucho & $12-13$ \\
\hline & & & Total & $14-15$ \\
\hline & & & & \\
\hline \multirow{5}{*}{2} & \multirow{5}{*}{ Sueño } & & Nulo & $5-7$ \\
\hline & & & Poco & $8-9$ \\
\hline & & & Moderado & $10-11$ \\
\hline & & & Mucho & $12-13$ \\
\hline & & & Total & $14-15$ \\
\hline \multirow{5}{*}{3} & \multirow{5}{*}{ Gastos } & & Nulo & $5-7$ \\
\hline & & & Poco & $8-9$ \\
\hline & & & Moderado & $10-11$ \\
\hline & & & Mucho & $12-13$ \\
\hline & & & Total & $14-15$ \\
\hline
\end{tabular}

\begin{tabular}{|c|c|c|c|c|}
\hline Id & POR DIMENSIÓN & PUNTOS OBTENIDOS & NIVEL DE ESTRÉS & $\begin{array}{l}\text { ESCALA DE } \\
\text { REFERENCIA }\end{array}$ \\
\hline \multirow{5}{*}{4} & \multirow{5}{*}{$\begin{array}{l}\text { Personal } \\
\text { Es la suma de los puntos obtenidos en } \\
\text { los Id. } 1,2 \text { y } 3 \text { (alimentación, sueño y } \\
\text { gastos) }\end{array}$} & & Nulo & $10-21$ \\
\hline & & & Poco & $22-27$ \\
\hline & & & Moderado & $28-33$ \\
\hline & & & Mucho & $34-39$ \\
\hline & & & Total & $40-45$ \\
\hline \multirow{6}{*}{5} & \multirow{6}{*}{ Interpersonal } & & & \\
\hline & & & Nulo & $0-7$ \\
\hline & & & Poco & $8-9$ \\
\hline & & & Moderado & $10-11$ \\
\hline & & & Mucho & $12-13$ \\
\hline & & & Total & $14-15$ \\
\hline & & & & \\
\hline \multirow{5}{*}{6} & \multirow{5}{*}{ Académico } & & Nulo & $0-7$ \\
\hline & & & Poco & $8-9$ \\
\hline & & & Moderado & $10-11$ \\
\hline & & & Mucho & $12-13$ \\
\hline & & & Total & $14-15$ \\
\hline
\end{tabular}

\begin{tabular}{|c|c|c|c|c|}
\hline Id & GENERAL & PUNTOS OBTENIDOS & NIVEL DE ESTRÉS & $\begin{array}{l}\text { ESCALA DE } \\
\text { REFERENCIA }\end{array}$ \\
\hline \multirow{5}{*}{7} & \multirow{5}{*}{$\begin{array}{l}\text { Es la suma de los puntos obtenidos en } \\
\text { los Id. 1, } 2,3,5 \text { y } 6 \\
\text { (alimentación, sueño, gastos, } \\
\text { interpersonal y académico) }\end{array}$} & & Nulo & $10-22$ \\
\hline & & & Poco & $23-35$ \\
\hline & & & Moderado & $36-48$ \\
\hline & & & Mucho & $49-62$ \\
\hline & & & Total & $63-75$ \\
\hline
\end{tabular}

Fuente: instrumento Estrigeree. 
No obstante, la interpretación de los resultados en los estudiantes no es menos importante puesto que permite evidenciar y analizar el potencial que el instrumento Estrigeree ofrece en los términos para los cuales fue desarrollado.

\section{g) Resultados por dimensiones}

En el Gráfico No.1 se muestran los diferentes resultados de las tres dimensiones; se pudo observar entonces que no existe algún nivel o factor estresante en las dimensiones académica e interpersonal en un $86.3 \%$ así como en el 74.4\% respectivamente. En la dimensión personal se observa que sólo el $9.8 \%$ refiere no haber algún factor estresante relacionado a dicho constructo, es decir, se puede interpretar que el área que tiene más relación con aquellos elementos que puedan generen estrés es la dimensión de los factores personales.

En la dimensión personal se puede observar que existen múltiples asociaciones a factores que puedan causar estrés puesto el $39.2 \%$ de la población refiere tener poco estrés, el 33.3\% menciona tener un estrés moderado, el $15.7 \%$ refiere una percepción de mucho estrés y el $2 \%$ lo relacionan a un estrés total. En el caso de la dimensión interpersonal, se puede observar que sólo el $20.6 \%$ de la población refiere tener algún grado de estrés y que aunque no menos importante, se representa la distribución en un $14.7 \%$ de poco estrés, el $4.9 \%$ refiere estrés moderado y sólo el $1 \%$ hace mención de mucho estrés. Ningún caso en esta dimensión presento estrés total.

Por otro lado, la dimensión académica demuestra aún menos presencia de factores estresantes puesto que el $10.8 \%$ de la población refiere poco estrés, otro $2.9 \%$ menciona tener estrés moderado. Lo que destaca en la dimensión es que no se presentaron casos en las categorías de mucho y total estrés; como antes ya comentado, el $86.3 \%$ de la población se concentran en la categoría de nulo estrés. 
Gráfico No. 1. Resultados por dimensiones.

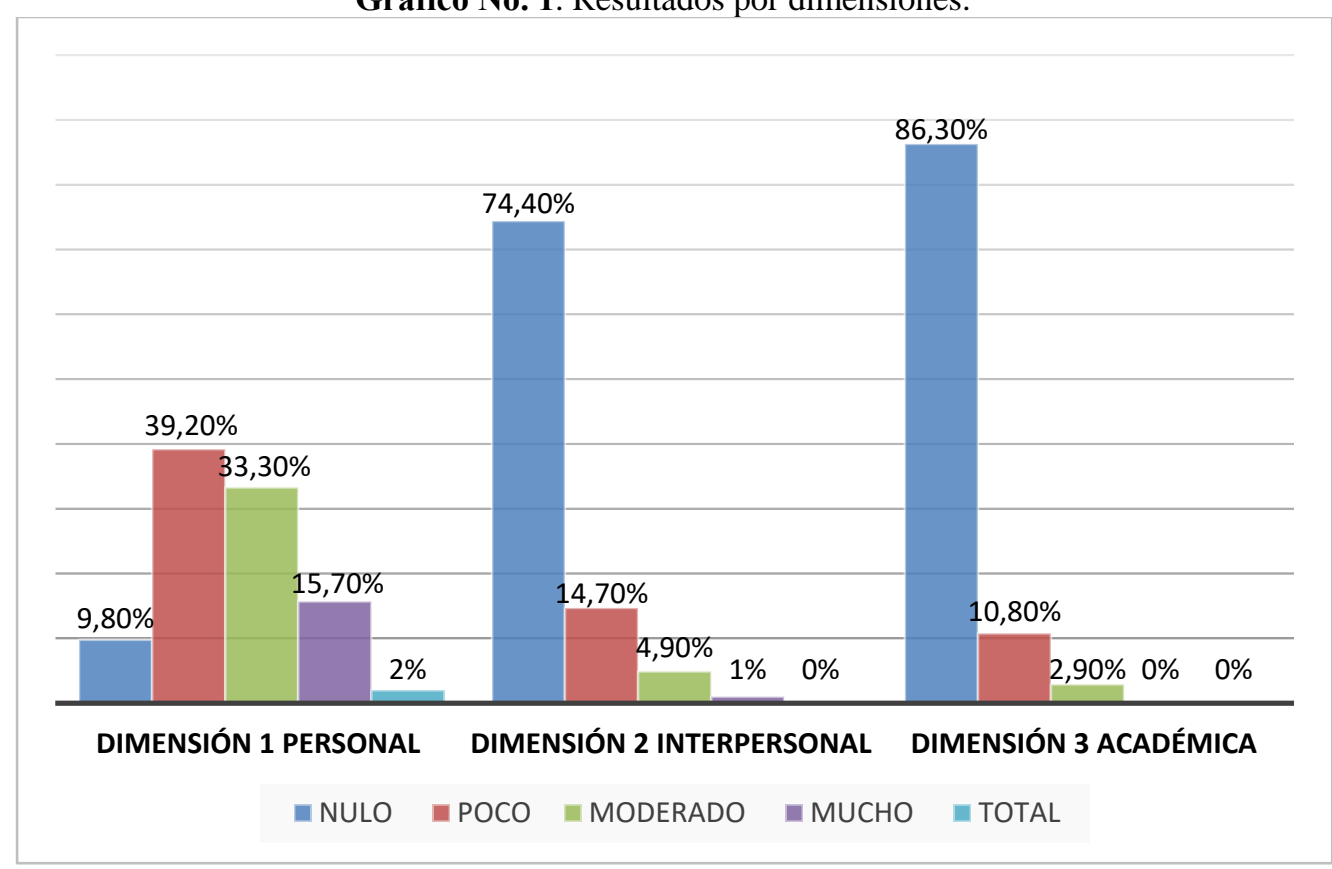

Fuente: instrumento Estrigeree.

\section{h) Resultados totales}

En la Gráfica No. 2 se puede observar una característica muy importante del instrumento que es poder medir en su totalidad el nivel de estrés en el caso de una autoaplicación de cada individuo, es decir, lo anterior con la finalidad de auscultar no sólo los factores involucrados, sino en qué medida estos tienen un impacto global en la persona desde la perspectiva de estrés. Por lo tanto, en términos de una evaluación global de la persona, sólo el 2.9\% de la población refiere no tener estrés o los factores involucrados no son lo suficientemente incidentes en la generación del mismo. El 34.3\% menciona contar con poco estrés. Por otro lado, el $52 \%$ demuestra tener un nivel de estrés moderado así como el $10.8 \%$ de los evaluados refieren mucho estrés. De la muestra se observó que ningún caso presentó un nivel de estrés total. 
Gráfico No. 2. Resultados totales.

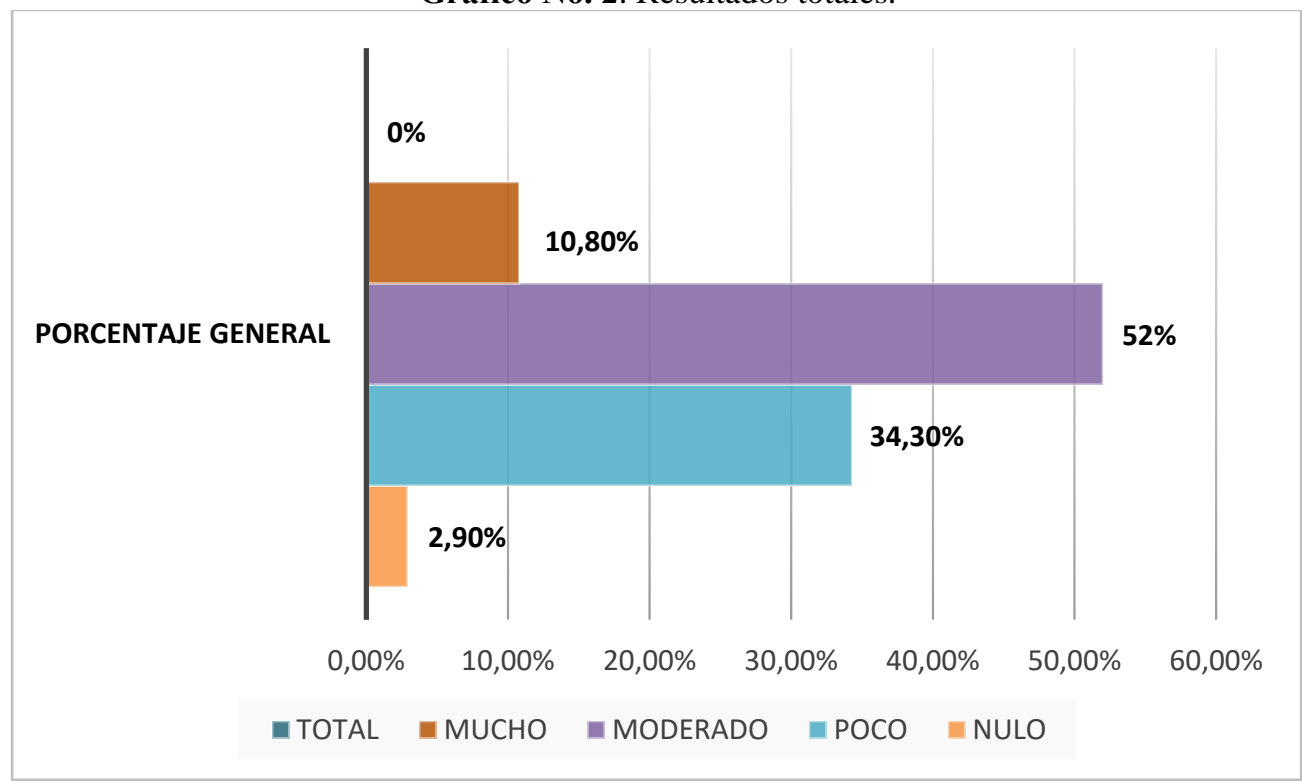

Fuente: instrumento Estrigeree.

\section{i) Resultados por sexo}

Como se muestra en la Gráfica No. 3, en el caso de los hombres se observa una concentración de factores estresantes relativamente bajo debido a que los resultados que se presenta en el rango de poco a mucho estrés se distinguen en en un 20.5\% en la dimensión interpersonal así como en un $24 \%$ en la dimensión académica. No tanto así en la dimensión personal que es en donde se muestra un $90.1 \%$ de presencia de estrés distribuidos en el $37.9 \%$ como poco estrés, el $25.50 \%$ en estrés moderado y el $24.7 \%$ con mucho estrés; por lo tanto se observa de igual forma que la dimensión personal es la que tiene mayor influencia en los elementos relacionado con la presencia de estrés. Es importante también observar que el $75.8 \%$ no refiere la presencia de estrés en la dimensión académica así como el $79.3 \%$ lo menciona nulo en la dimensión interpersonal. Por último, es también sustancial verificar que en ninguna dimensión se presentó algún caso de estrés total. 
Gráfico No. 3. Resultados por sexo masculino.

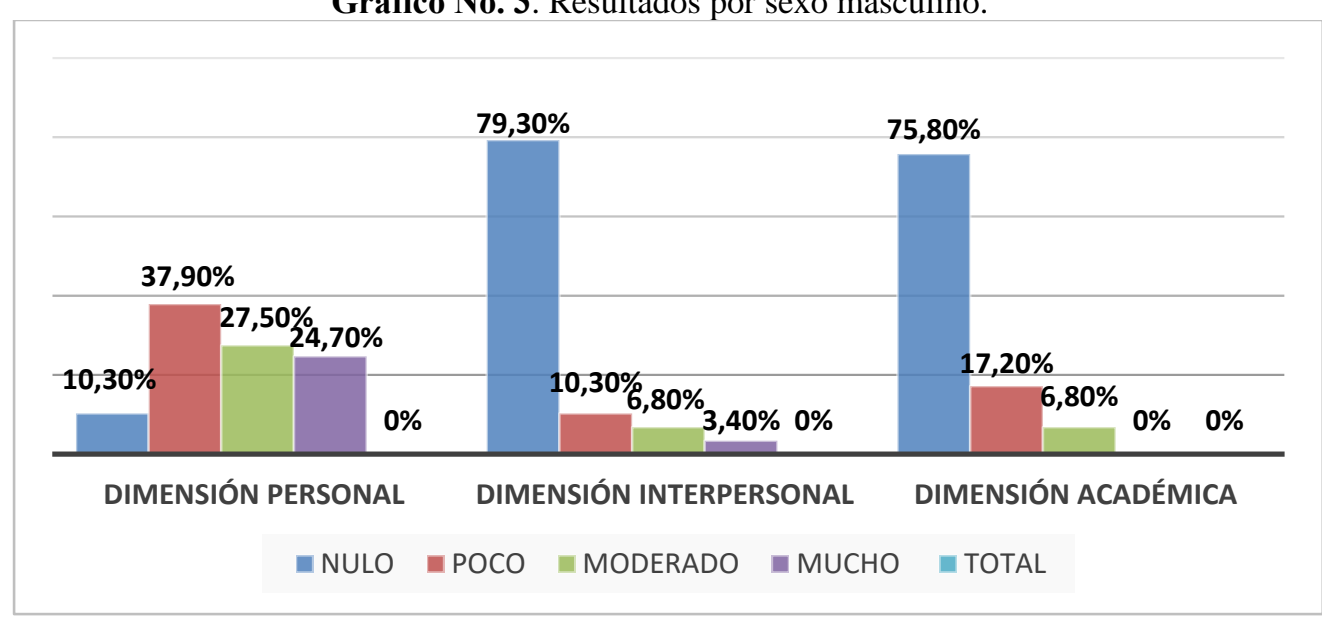

Fuente: instrumento Estrigeree.

En la Gráfica No. 4 se demuestra al igual que en los hombres un estrés bajo, siendo más notorio en la dimensión académica con un porcentaje de 86.67 en el rubro nulo así mismo en la dimensión interpersonal con un porcentaje de $79.50 \%$.

De igual manera que en el caso de los hombres, la dimensión que se encuentra afectada y en la cual los alumnos notaron al instante de la realización de su encuesta fue en la dimensión académica mostrando los siguientes porcentajes: $9.5 \%$ en como calificación nula, $41.09 \%$ en estrés poco, siendo éste rubro con mayor porcentaje de la dimensión personal, un $35.6 \%$ en un estrés moderado, mucho estrés con el $12.32 \%$ y un $1.36 \%$ porcentaje en estrés total, arrojando en estos resultados mayor atención en la presente dimensión.

Gráfico No. 4. Resultados por sexo femenino.

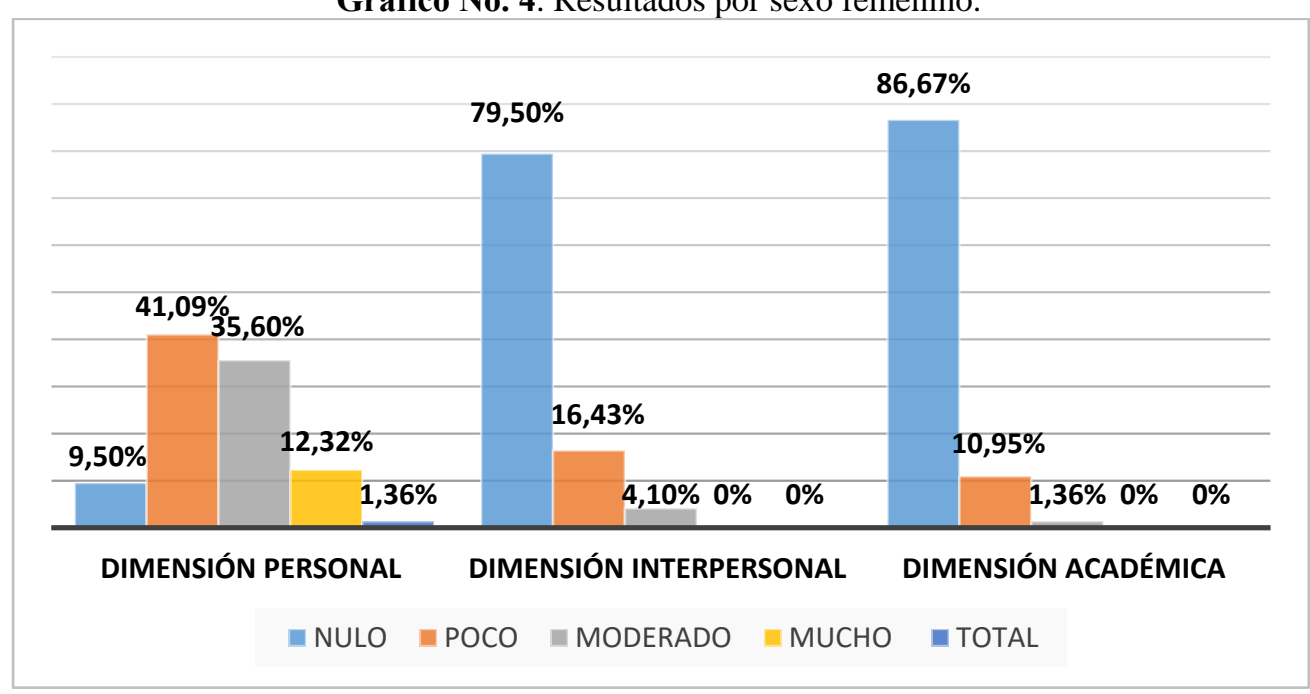

Fuente: instrumento Estrigeree. 


\section{Conclusión:}

Después de una exhaustiva y detallada metodología de trabajo, se concluye que se logra el objetivo de estudio debido a que el instrumento es válido y confiable, se considera también que es estable es su medición temporal corroborado por el intervalo de su aplicación y sus resultados del alpha de Cronbach. Por otro lado, desde la perspectiva de manejo físico y visual se determina que es claro, sistemático y completo, de rápida aplicación y procesamiento. Finalmente, el instrumento cumple con su objetivo de diseño.

\section{Discusión:}

Un estudio sobre la ansiedad define el estrés como una reacción del organismo frente a las demandas del entorno. Desde entonces se ha conceptualizado de distintas formas como respuesta, como estímulo o como proceso, considerando que el estrés es una condición de salud que afecta a gran parte de la sociedad debido a las actividades de la vida diaria, las exigencias escolares, laborales y las nuevas tecnologías, etc. (Comín, De la Fuente y Gracia, 2011).

En este estudio se pudo observar que el estrés puede representar una problemática en las áreas personal y académica y no así en los factores interpersonales, por lo que se puede asumir que tiene un impacto a nivel académico en el desarrollo de las prácticas clínica en fisioterapia tal cual se demuestra en el estudio "Influencia del estrés en el rendimiento académico de un grupo de estudiantes universitarios" de Domínguez, Domínguez y Guerrero (2015), en donde concluyen que es indispensable poner atención en este problema en la educación, pues es en este ámbito donde se encuentran casos de ansiedad por trabajos escolares, exámenes parciales, exámenes finales, así como también existe la presión de trabajos de equipo, el acoplamiento a diversos ambientes de trabajo, entre otros.

También se determinó con base en los resultados que en la dimensión personal, los factores tales como la alimentación, el sueño y los gastos económicos son aquellos indicadores que potencialmente pueden generar riesgo de desarrollar estrés en los estudiantes, así como en la dimensión académica que en donde particularmente el sexo masculino arrojó un elevado nivel de estrés.

Esta investigación abarcó diversos aspectos interdisciplinares que contribuyeron para que el instrumento diseñado Estrigeere, cumpliera con los requisitos necesarios para poder evaluar el nivel de estrés del alumno en prácticas clínicas de fisioterapia.

La creación de dicho instrumento permitirá que las instituciones educativas y aquellas donde se realizan prácticas clínicas de fisioterapia evalúen los impactos que genera el estrés en los estudiantes, relacionado a su 
calidad de vida, con la finalidad de mejorar las condiciones para el aprendizaje y el desarrollo profesional.

\section{References:}

1. Alfonso, B., Calcines, M., Monteagudo, R. y Nieves, Z. (2015). Estrés Académico. Cuba. Edy Me Centro, (2)163-178.

2. Baptista, M., Hernández, R., \& Fernández, C. (2014). Metodología de la investigación. México; Mc Graw Hill. $6^{\mathrm{a}}$ Ed.

3. Castillo I, Orozco Y \& Alvis. (2015). Síndrome de Burnout en el personal médico de una institución prestadora de servicios de salud. Revista industrial de salud.

4. Comín, E., De la Fuente, I., \& Gracia, A. (2011). El estrés y el riesgo para la salud. España: Departamento de prevención. $11^{\circ} \mathrm{Ed}$.

5. Corral, Y. (2009). Validez y confiabilidad de los instrumentos de investigación para la recolección de datos. Facultad de ciencias Económicas y Sociales, Universidad de Carabobo Valencia, Estado de Carabobo Venezuela. Pp. 228-247.

6. Domínguez, R., Domínguez, J.,y Guerrero, G. (2015). Influencia del estrés en el rendimiento académico de un grupo de estudiantes universitarios. Educación y ciencia, 4(43), 31-40.

7. Garbanzo, G. (2007). Factores asociados al rendimiento académico en estudiantes universitarios, una reflexión desde la calidad de la educación superior pública. Rev. Educación, 31(1) 43-63.

8. García, R.; Natividad, L. Pérez, F. \& Pérez, J. (2011). Evaluación del estrés académico en estudiantes de nueva incorporación a la universidad. Departamento de psicología Evolutiva y de la Educación. P.P. 143-.154.

9. George, D. y Mallery, P. (2003). spss for Windows step by step: A Simple Guide and Reference. 11.0 Update (4. ${ }^{\mathrm{a}}$ ed.). Boston: Allyn \& Bacon.

10. Gómez C, Puga A, Mayan J \& Gandoy M. (2012). Niveles de estrés en el personal de enfermería de unidades de cuidados paliativos. Escuela Universitaria de Enfermería. Santiago de Compostela España.

11. Gutiérrez, N., Becerra, J., Martínez, M. \& García, M. (2017). Efectos de la meditación sobre el estrés académico en estudiantes de licenciatura en fisioterapia. European Scientific Journal. Vol. 13, No. 19, ISSN: $1857-7881$.

12. Lewis, A. (2003). Test Psicológicos y evaluación. Pearson educación; México.

13. Martínez, A. (2010). El síndrome de Burnout. Evolución conceptual y estado actual de la cuestión. Rev. Vivat academia, 112, 1-40. Recuperado el 8 de Noviembre del 2016 de: 
http://pendientedemigracion.ucm.es/info/vivataca/numeros/n112/PDF s/Anbelacop.pdf.

14. Rodríguez, M., Sanmiguel, M., Muñoz \& Rodríguez C. (2014). El estrés en estudiantes de medicina al inicio y final de su formación académica. Revista Iberoamericana de Educación.

15. Rosales, Y. (2012). Estudio Unidimensional del Síndrome de Bornaut en Estudiantes de Medicina de Holguín. Cuba. Rev. Asoc. Esp. Neuropsiq. Vol.32 no. 116.

16. Tamayo V, Ortiz Y \& Giraldo V. (2014). Las prácticas formativas en el programa de fisioterapia universidad CES. Revista CES Movimiento y Salud Vol. 2

17. World Confederation for Physical Theraphy (2011). Recuperado 2016. 